\title{
A Feature Similarity-Based Multi-Resolution Method for Image Registration
}

\author{
Zhihui Wang ${ }^{1, *}$, Yuzhu Xie ${ }^{1,2}$, Yuejiao Fan ${ }^{1}$, Wenbo $\mathrm{Xu}^{1}$, Qila Sa ${ }^{1}$ and Jinlin $\mathrm{Li}^{1}$ \\ ${ }^{1}$ Dept of Electronic Engineering, College of Electronic Information and Engineering, \\ Inner Mongolia University, Hohhot 010021, China \\ ${ }^{2}$ China Mobile Group Neimenggu Company Limited, Hohhot 010000, China \\ ${ }^{*}$ Corresponding author
}

\begin{abstract}
We present a method to automatically register images based on feature similarity and image decomposing by multiple wavelet-transform. We use GHM multi-wavelet to decompose the image, then use mean-shift image segmentation algorithm to extract the image feature. We serve the regional features as matching primitives, and put forward an improved image registration method based on invariant descriptor. The experimental results show that the algorithm in reducing computational complexity and enhance the algorithm's robustness can get better effect.
\end{abstract}

Keywords-image registration; GHM multi-wavelet; feature extraction; feature similarity; invariant descriptor

\section{INTRODUCTION}

Image registration is one of the important steps in actual problems such as recognition of target, detection of image properties difference and fusion of multi-source information [1]. Image registration is the matching process of two or more images. Those images, which may be taken from different sensors, at different time or from different perspectives of the same scene, contain different information. Image registration has been widely used in military, medical science, aerospace and other important scientific research field. Currently image registration has made certain achievements in identification of military target, detection of image difference, diagnosis of tumor, fusion of remote sensed images, etc [2]. So the research to make image registration faster and more efficient is very necessary.

Image registration is a combination of the following elements [3]:

Feature space: The characteristics which are extracted from reference images and floating images can be used for the image registration;

Search space: The space is made up of the range and the way of the process of image transformation;

Search strategy: Finding the optimal registration parameters in the search space, the parameters are based on the value of similarity metrics in the process of search;

Similarity metrics: evaluate the advantage and disadvantage of each transformation results, and provide the basis for the next step of search strategy.
Commonly, image registration methods can be divided into the main three categories: Image registration method based on gray level [4], image registration method based on feature [5] and image registration method based on the model [6]. The biggest characteristic of image registration method based on gray level is that the whole image pixels are directly involved in the process of image registration [7]. This method cannot lost any image information, so the effect of the method can get higher precision. But the method cannot avoid large amount of calculation and poor real-time performance. Image registration method based on feature is to extract features which can represent the nature of the images for registration, so that this method can get relatively small amount of calculation and high real-time performance. Image registration method based on model mainly use nonlinear calibration to register image. The registration is based on the establishment of mathematical model for image distortion. This method is mostly used in medical image registration [8].

In recent years, more and more scholars pay great attention to the theory of wavelet transform[9-10]. To achieve higher efficiency and precision of registration, scholars try to combine image registration with wavelet transform. And a lot of experiments have confirmed the feasibility of these combination algorithms [11-12].

This paper applies multi-wavelet transform to image registration method based on feature, and puts forward a new similarity measure standard. The simulation experimental results show that the algorithm could get good effectiveness.

\section{MULTI-WAVELET TRANSFORMATION OF IMAGE}

\section{A. GHM Multi-Wavelet System}

The development of multi-wavelet is on the basis of the MRA (Multi-resolution analysis). Multi-wavelet expands the space of multi-scale analysis which is generated by two or more scaling functions. Multi-wavelet retains wavelet's unique time-frequency localization characteristic, but also overcomes the defect of wavelet. It realizes smoothness, tight branch, symmetry and orthogonality all together, which are very important characters in the practical application [13]. 


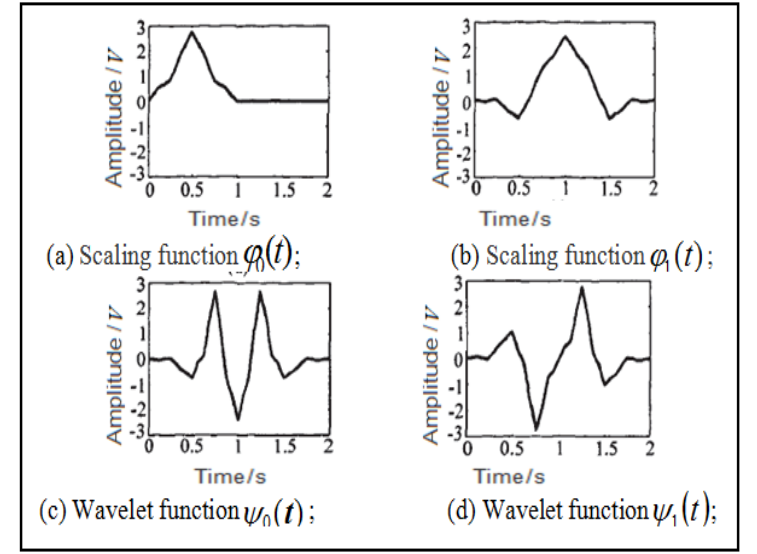

FIGURE I. GHM MULTI-WAVELET

In multi-wavelet, assuming multi-scaling function and multi-wavelet function as $\Phi(t)=\left[\phi_{0}(t), \phi_{1}(t), \ldots \phi_{r-1}(t)\right]^{\tau}$ and $\psi(t)=\left\{\psi_{0}(t), \psi_{1}(t), \ldots, \psi_{r-1}(t)\right\}^{T}$.

$$
\left\{\begin{array}{l}
\Phi(t)=\sum_{k=0}^{m-1} H_{k} \Phi(2 t-k) \\
\Psi(t)=\sum_{k=0}^{m-1} G_{k} \Phi(2 t-k)
\end{array}\right.
$$

The above two equations constitute two scale function. Where: $H_{k}$ and $G_{k}$ are respectively $r \times r$ coefficient matrix of low-pass and high-pass filter.

This paper use GHM multi-wavelet to decompose images for one layer. The process of decomposition by GHM is the same as the process described in section 2.2 of this paper. The GHM multi-wavelet is one of the orthogonal multi-wavelet. It is put forward by Geronimo, Hardin and has advantage of orthogonality, symmetry/inverse symmetry, compact support and 2 order of approximation order. The detail analysis of GHM multi-wavelet is described in the reference paper [14]. Two multi-scaling functions and multi-wavelet functions of GHM are shown in Figure I, when $\mathrm{K}=2, \mathrm{~L}=4$.

\section{B. Multi-Wavelet Transformation of Image}

Multi-wavelet transform of the image essentially refers to the discrete multi-wavelet transform of discrete data. Before the transform, filter the rows' and columns' discrete data respectively to transform the image information into a vector signal. This step is often called pre-filtering. There will be $k^{2}(3 L+1)$ sub-images if multi-wavelet decompose the image to $L$ layers, $k$ is the number of wavelet function. After decomposition of image, each layer contains high frequency and low frequency images. Among them, the high frequency sub-image contains the detail of the image information, and the low frequency sub-image contains most of the original image information. The specific process of image decomposition is described in the reference paper [15]. Figure II shows three layers decomposition process of multi-wavelet, where $k=2$.

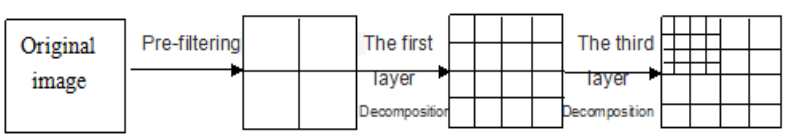

FIGURE II. PROCESS OF MULTI-WAVELET DECOMPOSITION

\begin{tabular}{|c|c|c|c|}
\hline $\mathrm{L}_{1} \mathrm{~L}_{1}$ & $\mathrm{~L}_{1} \mathrm{~L}_{2}$ & $\mathrm{~L}_{1} \mathrm{H}_{4}$ & $\mathrm{~L}_{1} \mathrm{H}_{2}$ \\
\hline $\mathrm{L}_{2} \mathrm{~L}_{1}$ & $\mathrm{~L}_{2} \mathrm{~L}_{2}$ & $\mathrm{~L}_{2} \mathrm{H}_{6}$ & $\mathrm{~L}_{2} \mathrm{H}_{2}$ \\
\hline $\mathrm{H}_{1} \mathrm{~L}_{2}$ & $\mathrm{H}_{3} \mathrm{~L}_{2}$ & $\mathrm{H}_{1} \mathrm{H}_{1}$ & $\mathrm{H}_{1} \mathrm{H}_{2}$ \\
\hline $\mathrm{H}_{2} \mathrm{~L}_{1}$ & $\mathrm{H}_{2} \mathrm{~L}_{2}$ & $\mathrm{H}_{2} \mathrm{H}_{4}$ & $\mathrm{H}_{2} \mathrm{H}_{2}$ \\
\hline
\end{tabular}

FIGURE III. THE FIRST LAYER OF MULTI-WAVELET DECOMPOSITION

After the first layer wavelet decomposition, the image is decomposed into 16 sub-images, including 12 high-frequency sub-images, and four low-frequency sub-images. Located in the upper left part of the image and contain low-frequency information, while the remaining part contains high-frequency information. 16 sub-images are shown in figure III.

\section{MethodOLOGY}

Feature-based image registration generally includes five steps [16]: preprocessing of image; feature extraction of image; features matching of image; calculation of transform model's parameters; coordination of transformation and interpolation. Among these steps, feature extraction and features matching are the most important parts.

Feature extraction refers that according to gray scale characteristics of the reference image and float image, using image segmentation technology and pattern recognition, etc to extract the steady feature of the image. The most common extraction of feature primitives are point, line and region. As matching primitives, the three types of feature primitives has its own specific characteristics, advantages, way of extraction and limitations. For accurate registration results, various factors should be taken into account in the choice of feature primitives.

Features matching refers that use a certain registration algorithm to establish the one to one correspondence relation of extracted feature points. The set of corresponding points often called the control point set, then transformation model can be obtained through the control point set. Describe this process in mathematical language as follows:

$F_{1}=\left\{f_{11}, f_{12}, \cdots, f_{1 N_{1}}\right\}$ and $F_{1}=\left\{f_{21}, f_{22}, \cdots, f_{2 N_{2}}\right\}$ are the feature point sets which are extracted from the reference image and float image, $N_{1}$ and $N_{2}$ are the number of the point. $\Gamma$ is the space for transformation model parameters. Feature matching is to find the optimal transformation model parameter $P, P \in \Gamma$, so as to get the biggest value of similarity between $P\left(F_{2}\right)$ and $F_{1}$. Process of feature matching is shown in figure IV. 


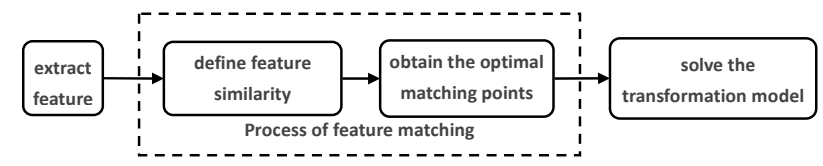

FIGURE IV. FLOW CHART OF FEATURE MATCHING PROCESS

As shown in figure IV, how to define similarity measure and obtain optimal matching points constitute the main part of matching of image features.

According to the different definition of similarity measure, feature-based image registration can be divided into two ways:(1) spatial relationship-based method. Definition of this method is based on spatial relations in a certain transformation models of matching primitives which are extracted from the reference image and float image; (2) feature similarity-based method. This method define the similarity measure by minimum distance between a certain invariant descriptors, which are used to describe the features of images.

Since most of registration algorithm contains a large amount of calculation, it is very necessary to search a good method to obtain optimal matching points. Commonly used optimization algorithms contain control points combined with least square method, the golden section method, Brent method and so on.

\section{A. Proposed Image Registration Algorithm Based on Feature Similarity}

This paper assumes that happened between reference image and float image is affine transformation.

- Firstly, images are decomposed by GHM multiwavelet.

- Secondly, for the low-frequency parts of decomposed images, regional feature extraction based on Meanshift algorithm is adopted to get regional features.

- Thirdly, using seven affine invariant moment as descriptors of regional features, we define a similarity measure based on the minimum distance criterion of affine invariant moment, and obtain initial matching regional features according to similarity measure.

- Finally, get control points set by using the exhaustive policy.

Combining with the least squares algorithm, we can estimate the optimal affine transform model parameters. The whole process of algorithm is shown in figure $\mathrm{V}$.

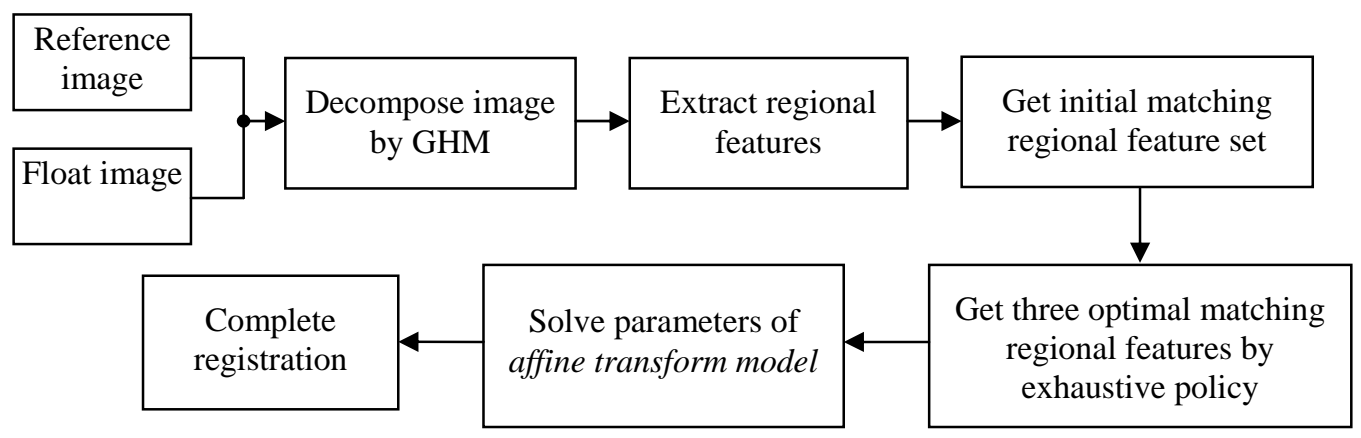

FIGURE V. FLOW CHART OF IMAGE REGISTRATION

\section{B. Image Feature Extraction}

This paper uses the method of regional feature extraction based on Mean-shift algorithm. Pick regional features among the low-frequency sub-images of decomposed images. Process of the extraction is shown in Figure VI.

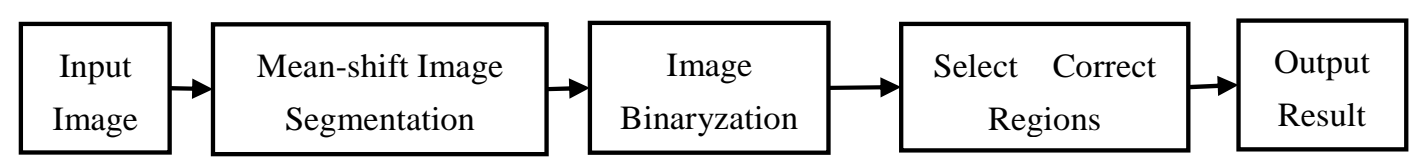

FIGURE VI. FLOW CHART OF IMAGE FEATURE EXTRACTION

It is observed from the figure above that Mean-shift algorithm is the key step of the extraction process. Mean-shift algorithm is a classical method of image segmentation. It is put forward by Fukunaga and Hostetler which is a kind of excellent method of nonparametric feature space analysis. Its essence belongs to the kernel density estimation. The detail analysis of Mean-shift algorithm and the concrete steps of Mean-Shift image segmentation algorithm are described in the reference [17]. Use the method of regional feature extraction towards the low-frequency sub-images of decomposed images. Assumes that the number of regional feature extraction from reference images is $R_{1}$, marked as $P_{1}=\left\{p_{1}^{i}, i=1,2, \cdots, R_{1}\right\}$, marked their coordinates of center of gravity as $M_{1}=\left\{\left(x_{1}^{i}, y_{1}^{i}\right), i=1,2, \cdots, R_{1}\right\}$; Similarly, assumes that the number of regional feature extraction from float images is $R_{2}$, marked as $P_{2}=\left\{p_{2}^{i}, i=1,2, \cdots, R_{2}\right\}$, marked their coordinates of center of gravity as $M_{2}=\left\{\left(x_{2}^{i}, y_{2}^{i}\right), i=1,2, \cdots, R_{2}\right\}$. 


\section{Matching of Regional Features}

After the extraction of regional features, we use the minimum distance algorithm of seven affine invariant moment to establish the corresponding feature matching strategy. So as to determine the matching corresponding relationship between the regional features. Affine invariant moment, which is used to described regional features, is one of the most commonly used invariant descriptors.

For each regional features, its seven affine invariant moment can be saw as a point in seven dimensions, so the similarity of any two regional features can be measured by the distance between any two points. Define a distance moment with the size of $R_{1} \times R_{2}$ to show the similarity between any two regional features, the distance moment is :

$$
\begin{gathered}
D=\left\{d_{i j}, i=1,2, \cdots, R_{1}, \quad j=1,2, \cdots, R_{2}\right\} \\
d_{i j}=\sqrt{\sum_{k=1}^{7}\left(\phi_{1}^{i}(k)-\phi_{2}^{j}(k)\right)^{2}}
\end{gathered}
$$

where $\phi_{1}^{i}(k)$ represents K-Order affine moment invariants of the $i$-th regional feature $\left(r_{1}^{i}\right)$ in reference image; $\phi_{2}^{j}(k)$ represents K-Order affine moment invariants of the $j$-th regional feature $\left(r_{2}^{j}\right)$ in float image.

In order to express the similarity of regional feature more intuitive, we define a similarity matrix as:

$$
C=\left\{c_{i j}, i=1,2, \cdots, R_{1}, \quad j=1,2, \cdots, R_{2}\right\}
$$

where $c_{i j}=1 / d_{i j}$. It can be found that the bigger $c_{i j}$ is, the more similar regional features are.

According to the minimum distance criterion of invariant descriptors, we obtain top six values from different rows and columns of the similarity matrix $C$. The top six values correspond to six pairs of regional features, which are possible matching features. The set of possible matching features is defined as $T$ :

$$
T=\left\{\left(l_{1}^{i_{r}}, l_{2}^{j_{s}}\right) \mid r=1,2 . \cdots, 6 ; s=1,2, \cdots, 6\right\}
$$

\section{Image Registration Algorithm}

In this paper, we use the exhaustion search strategy to pick the three optimal matching features from the set $T$ which is gotten from previous section, and use the three matching features' center of gravity point. Combined with the least squares algorithm, the optimal affine transform model parameters can be estimated. The detailed process of matching algorithm is as follows:
- $\quad$ Step 1: According to similarity measure, choose the top six regional feature pairs as initial matching regional features from similarity matrix $C$, which is calculated by (4).

- $\quad$ Step 2: Select three optimal matching features using the exhaustive policy from set $T$. Specific operations are as follows:

1) From the set $T$, three pairs of matching features in different rows and different columns are randomly selected, and use their center gravity coordinates to calculate the value of affine transform model parameter.

2) For $j=1,2, \cdots, 6$, take the center gravity coordinates of float image's regional feature $l_{2}^{j}$ into acquired affine transform model, which will get a point $\mathrm{G}$. Then finding out a regional feature $r_{1}^{i}$ from $P_{1}$, which is the most closed to $G$ point. Assuming $n$ is the count of regional feature pairs that meet the requirement $d=\left\|G-M_{1}^{i}\right\|<V$. ( $\|\cdot\|$ means Euclidean distance, $V$ means threshold).

3) Repeating 1) and 2), until have no regional feature can meet the requirements, and the calculation will end. Then, pick three regional feature pairs as the most optimal matching features pairs when the value of $n$ gets maximum.

- $\quad$ Step 3: The affine transformation model parameters values can be get by computing center gravity coordinates of regional feature pairs form step 2 .

Feature extraction effect is relevant to the value of threshold, which is in direct proportion. And in actual operation, the optimal value of $V$ is yet decided by the actual situation of image and feature.

\section{IMPLEMENTATION OF IMAGE REGISTRATION ALGORITHM}

\section{A. Experimental Results}

In this paper, we use MATLAB software to simulate the proposed algorithm. The specific results are as follows:

The results of image decomposition based on GHM multiwavelet for one layer is shown in figure VII. Figure VII (1) and (2) are the original reference image and float image, Figure VII.(3) and (4) are respectively the decomposed images of (1) and (2).

The result of the feature extraction by using the method of regional feature extraction based on Mean-shift segmentation algorithm is shown in figure VIII. Figure VIII.(1) and (2) are the result of extraction towards the original reference image and the float image, Figure VIII (3) and (4) are respectively the result of extraction towards the decomposed images. 


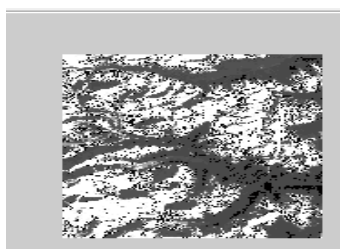

(1)

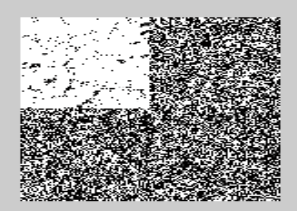

(3)

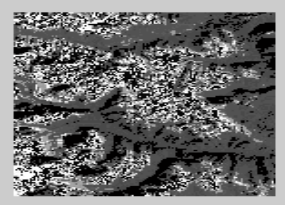

(2)

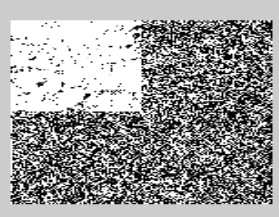

(4)

FIGURE VII. IMAGE DECOMPOSITION BASED ON GHM MULTIWAVELET

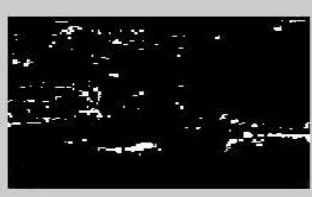

(1)

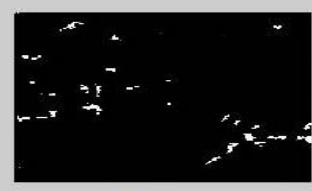

(3)

FIGURE VIII. IMAGE FEATURE EXTRACTION
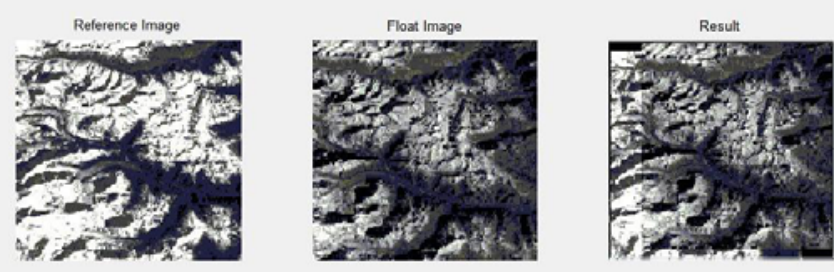

FIGURE IX. IMAGE REGISTRATION RESULT

The result of the final registration is shown in figure IX.

\section{B. Analysis of Performance of Proposed Algorithm}

We analyze the proposed method in this paper form the aspect of efficiency, robustness, and adaptability as follows.

1) Efficiency: According to Figure VIII, because of the feature extraction is only towards the low-frequency subimages of decomposed images, the number of features which are extracted from reference image and float image decrease obviously. So the step of GHM multi-wavelet decomposition decreases calculation and matching time. Otherwise, even though using exhaustion search strategy, the proposed matching algorithm still keeps a low complexity, because of the set $T$ which has only six feature pairs in it. In conclusion, this method improves performance of matching image significantly.

2) Robustness: As we all know, many factors, such as light, temperature sensors and transmission channel, will bring huge image noise which are mostly concentrated in the high frequency part of the image. The method which extract regional features from low-frequency sub-images of decomposed images can effectively avoid the disturbance of noise. It also makes the extracted features more precise, and ensures the accurateness of following process. What's more, appropriate increase of possible matching region pairs can increase matching accuracy.

3) Adaptability: Although this paper assumes what happened between reference image and float image is affine transformation, it can be obviously seen that the registration algorithm in this paper can be easily extended to other situations, which is not subject to the type of feature extraction and the transformation model.

\section{CONCLUSIONS}

A method of applying GHM multi-wavelet transform to image registration method based on feature similarity is put forward. The basic process of image registration and the advantages of multi-wavelet are briefly described in this paper. We also introduce the process of image decomposition based on GHM multi-wavelet. Specific steps and performance of the method are analyzed. Simulation experimental results show that the method proposed in this paper can get good effectiveness.

\section{ACKNOWLEDGMENT}

The research in this paper was sponsored by the National Natural Science Foundation of China (Grant No.61261003), and the Natural Science Foundation of Inner Mongolia Autonomous Region of China (Grant No.2015MS0610).

\section{REFERENCES}

[1] Haili Huo. "Research on Key Algorithms of image registration", BEIJING INSTITUTE OFTECHNOLOGY, 2015.

[2] G.S. Madhuri, M.P. I. Gandhi. "Image registration quality assessment with similarity measures-A research study", 2015 IEEE International Conference on Computational Intelligence and Computing Research (ICCIC), 2015.

[3] E.J.M. Rignot, R. Kowk, J.C. Curlander, S. Pang Automated. "Multisensor registration requirements and techniques": Photogrammetric Engineering and Remote Sensing, 57 , pp. 1029-1038, 1991.

[4] Cardinal Marie-Hélène Roy , Meunier Jean, Soulez Gilles, Maurice Roch L, Therasse Eric, Cloutier Guy. "Intravascular ultrasound image segmentation: a three-dimensional fast-marching method based on gray level distributions", IEEE Transactions on Medical Imaging,Vol.25 (5), pp.590-601, 2006.

[5] H. Li, B. S. Manjunath, S. K. Mitra. "A contour-based approach to multisensor image registration", IEEE Transactions on Image Processing, 4, pp. 320-334, 1995.

[6] Bonmassar G, Schwartz E.L. Improved cross-correlation for template maching on the Laplacian Pyramid[J].Pattern Recognition., 19(8), pp. 763-770. 1993. 
[7] LU Kai, LI Cheng-jin, ZHAO Xun-jie, ZOU Wei, ZHANG Xue-song. " A Fast Sub-pixel Image Registration Algorithm ", Infrared Technology, vol. 35, pp. 27-30, 2013.

[8] LU Xue-Song, TU Sheng-Xian, ZHANG Su. "A Metric Method Using Multidimensional Features for Nonrigid Registration of Medical Images", ACTA AUTOMATICA SINICA, vol. 42, pp. 1-8, 2016.

[9] Kumar N A M, Sathidevi P S. "Image match using wavelet-Coloue SIFT features", The $7^{\text {th }}$ IEEE Conference on Industrial and Information System (ICIIS), pp. 1-6, 2012.

[10] Yiquan Wu, Feixiang Tao, Zhaoqing Cao."Iamge registration algorithm based on dual tree complex wavelet transform and SURF", Systems Engineering and Electronics, vol. 36, pp. 997-1003, 2014.

[11] Fangchao Yu. "Iamge Registration Method Based on Non-seperable Wavelet Decomposition", Hubei University, 2014.

[12] Chao Ding. "Research and Application of Wavelet Transform in Twosource Remote Sensing Image Registration", Zhejiang University of Technology, 2015.

[13] C. K. Chui and J. A. Lian, "A Study of Orthonormal Mul-tiwavelets," Applied Numerical Mathematics, vol. 20, pp. 273-298, 1996.

[14] Geronimo J S, Hardin D P, Massopust P R. "Fraetal functions and wavelet expansions based on several scaling functions", J of Approx Theory, vol. 78, pp. 373-401, 1994.

[15] Ling Wang, Guo-xiang Song."The Pre-Processing of Multi-wavelet and Its Application in Image Compression", ACTA ELECTRONIC SINICA,vol. 10, pp. 1418-1420, 2001.

[16] B.Zitova and J.Flusser. "Image registration methods: A survey", Image and Vision Computing, vol. 21, pp. 977-1000, 2003.

[17] D.Comaniciu, P.Meer. "Mean shift: a robust approach toward feature space analysis", IEEE Transactions on Pattern Analysis and Machine Intelligence, vol. 24(5), pp. 603-619, 2002. 